\title{
Phenotyping bronchiectasis: is it all about sputum and infection?
}

\author{
Daniel J. Smith ${ }^{1,2}$ \\ Affiliations: ${ }^{1}$ The Prince Charles Hospital - Thoracic Medicine, Brisbane, Australia. ${ }^{2}$ QIMR Berghofer Medical \\ Research Institute - Lung Inflammation and Infection Laboratory, Herston, Australia. \\ Correspondence: Daniel J. Smith, The Prince Charles Hospital - Thoracic Medicine, Rode Road, Chermside, \\ Brisbane, Queensland 4032, Australia. E-mail: daniel.smithवqqimr.edu.au
}

@ERSpublications

New subclassification of bronchiectasis patients may help inform targeted therapy

http://ow.ly/XT5ez

Bronchiectasis is the pathological end-point of a heterogeneous group of disease processes, defined by irreversible dilatation and distortion of the bronchi, which are chronically inflamed and commonly host to chronic bacterial infection. A search for an underlying cause, which may be amenable to a targeted intervention to prevent ongoing damage, is paramount but often fruitless, and the focus of therapy rapidly turns to empiric treatments to prevent infective exacerbations and retard disease progression.

There is, however, no licensed therapy specific to bronchiectasis and a paucity of high-level evidence to support the use of therapies including airway clearance, mucolytics and maintenance antibiotics, which are commonly employed to manage daily symptoms and acute exacerbations. The British Thoracic Society bronchiectasis management guidelines provide an in-depth summary of the available literature and are an excellent tool for guiding treatment decision making. However, they do not provide guidance on which patients are most likely to benefit from specific interventions [1].

The decision to commence a patient on a particular course of treatment, in general, is driven by perceived disease severity, likelihood of progression and symptomatic burden of disease.

Disease severity in bronchiectasis is hard to define. Radiological severity grading scores exist; however, there is often a disconnect between radiological severity, symptom burden and disease progression $[2,3]$. In 2014, competing bronchiectasis severity scores were published (FACED and the Bronchiectasis Severity Index (BSI)) [4, 5]. In each of these, a combination of patient demographics, symptom scores, comorbidities, and clinical, radiological and microbiological parameters were used to construct scoring systems, which, in the case of the BSI, predicted future mortality, and in the case of FACED, extended to prediction of future exacerbation frequency, hospitalisation and quality of life. Whilst these severity scores have utility in identifying an individual's risk of disease progression to a predefined outcome, they are of limited usefulness in guiding the implementation of interventions that may retard disease progression.

As an extreme example, employing the BSI (www.bronchiectasisseverity.com), a young patient with fewer than three lobes affected by bronchiectasis, who suffers with frequent exacerbations and hospitalisations, and is chronically infected with Pseudomonas and other bacterial pathogens, would obtain a similar disease severity rating as an underweight 70 year old with more than three lobes involved but no chronic bacterial infections, exacerbations or hospitalisations. Despite the similar scores (and consequently, predicted 4-year mortality), few would argue that these two very different clinical scenarios should be managed in a similar fashion.

Received: Jan 212016 | Accepted: Jan 222016

Conflict of interest: None declared.

Copyright @ERS 2016 
Consequently, it may be argued that phenotyping techniques, which focus less on pre-defined outcomes and more on factors that influence disease behaviour, have greater utility in identifying subjects who may respond to targeted therapies.

The FACED and BSI were each developed using multivariate analysis, with variables selected on the basis of their relatedness to the outcome of interest in an initial univariate analysis (e.g. mortality). Principal component analysis (PCA) and cluster analyses offer powerful analytical tools, which allow multiple nondependent variables to be filtered in order to identify a limited number of factors that most completely reflect the variability within a heterogeneous population, without the need for a priori determination of an important outcome. PCA analyses have recently been employed with promising results in the study of chronic obstructive pulmonary disease to identify phenotypically distinct groups with variable mortality, independent of forced expiratory volume in $1 \mathrm{~s}$ and Global Initiative for Chronic Obstructive Lung Disease severity $[6,7]$.

In this issue of the European Respiratory Journal, AliBERTi et al. [8] draw together data from five European bronchiectasis registries, and employ PCA and cluster analysis to identify subgroups of patients with distinct clinical and biological phenotypes.

Four distinct clusters are identified: chronic "Pseudomonas" infection, "other chronic infection", "daily sputum production" and "dry bronchiectasis". Disease burden, overall, decremented across these four clusters, with the Pseudomonas cluster experiencing the most severe symptoms, reduced quality of life, more rapid disease progression and increased 3-year mortality. Patients in the Pseudomonas cluster were more likely to have been prescribed maintenance macrolides and inhaled antibiotics, while patients within the dry bronchiectasis cluster experienced a more benign clinical course and were only rarely prescribed maintenance antibiotics.

Prospective recruitment of an external validation group was subsequently performed in a case-control manner to ensure recruitment of equal number of patients in each of the four identified clusters. Subjects in this cohort underwent sputum analysis for markers of neutrophilic inflammation. The clinical characteristics of the patients in each cluster accurately reflected those displayed in the derivation population and in addition patients in the "Pseudomonas" and "other chronic infection" groups displayed increased levels of sputum markers of neutrophilic inflammation than seen in the other two clusters.

On the surface, it may be argued that these findings do nothing more than confirm what is already known, that patients with bronchiectasis and chronic Pseudomonas infection suffer the greatest morbidity and those without symptoms follow a more benign course $[4,9,10]$. So how may the subgroups identified by AliberTi et al. [8] be used in the advancement of bronchiectasis care? In the clinic, the results may potentially be used as a method of triage, with "Pseudomonas-infected" patients given priority for early review and intensive follow-up, while "dry" patients may be seen infrequently or even receive nonspecialist follow-up. In the clinical trial setting, these clusters may be used to identify target populations for the recruitment of those most likely to respond to a specific intervention, thereby limiting the number of subjects required to demonstrate benefit (or harm).

It is, however, on the laboratory benchtop where the clusters described may prove to be of greatest influence, as the phenotypic groups described highlight "bigger picture" questions that are currently unanswered, including, why do some patients become infected with Pseudomonas while others (presumably with similar environmental exposures) do not? Why do some patients without clinically detectable infection produce sputum and others not? Does the presence of sputum indicate nonculturable (occult) infection? And, does structural lung disease in the absence of infection progress over time? It may be that the clusters identified simply reflect stepping stones along a common pathway, with patients starting initially with structural lung disease in the absence of symptoms, subsequently develop chronic sputum production and finally acquire chronic bacterial infection. More intriguing, is the possibility that each cluster represents a distinct clinical entity, with unique underlying biological mechanisms resulting in disease. Aliberti et al. [8] highlight differences in neutrophilic inflammation between infected and uninfected subgroups; however, this probably reflects a response to the infective insult as opposed to a marker of increased susceptibility to infection. Interrogation of how inflammatory and immune defence pathways differ between these distinct clinical phenotypes may begin to answer critical questions around susceptibility to infection. Indeed, it should be anticipated that future iterations or such phenotyping projects would incorporate systemic inflammatory markers, immunophenotyping, culture-independent microbiome profiling and other "omic" data to delineate differing disease endotypes.

The litany of question marks that punctuate this editorial reflect the uncertainty that still surrounds the aetiology and best practice in the management of bronchiectasis. However, the work of AliberTi et al. [8], is in an important step in subclassifying this heterogeneous group of patients in a manner that may pave the way to future mechanistic studies, which explain how these different disease phenotypes arise and inform the development of targeted therapeutics. 


\section{References}

1 Pasteur MC, Bilton D, Hill AT, et al. British Thoracic Society guideline for non-CF bronchiectasis. Thorax 2010; 65: Suppl. 1, i1-i58.

2 Eshed I, Minski I, Katz R, et al. Bronchiectasis: correlation of high-resolution CT findings with health-related quality of life. Clin Radiol 2007; 62: 152-159.

3 Reiff DB, Wells AU, Carr DH, et al. CT findings in bronchiectasis: limited value in distinguishing between idiopathic and specific types. AJR Am J Roentgenol 1995; 165: 261-267.

4 Chalmers JD, Goeminne P, Aliberti S, et al. The Bronchiectasis Severity Index. An international derivation and validation study. Am J Respir Crit Care Med 2014; 189: 576-585.

5 Martinez-Garcia MA, de Gracia J, Vendrell Relat M, et al. Multidimensional approach to non-cystic fibrosis bronchiectasis: the FACED score. Eur Respir J 2014; 43: 1357-1367.

6 Burgel PR, Paillasseur JL, Caillaud D, et al. Clinical COPD phenotypes: a novel approach using principal component and cluster analyses. Eur Respir J 2010; 36: 531-539.

7 Burgel PR, Roche N, Paillasseur JL, et al. Clinical COPD phenotypes identified by cluster analysis: validation with mortality. Eur Respir J 2012; 40: 495-496.

8 Aliberti S, Lonni S, Dore S, et al. Clinical phenotypes in adult patients with bronchiectasis. Eur Respir J 2016; 47: $1113-1122$.

9 Davies G, Wells AU, Doffman S, et al. The effect of Pseudomonas aeruginosa on pulmonary function in patients with bronchiectasis. Eur Respir J 2006; 28: 974-979.

10 Finch S, McDonnell MJ, Abo-Leyah $\mathrm{H}$, et al. A comprehensive analysis of the impact of Pseudomonas aeruginosa colonization on prognosis in adult bronchiectasis. Ann Am Thorac Soc 2015; 12: 1602-1611. 